\title{
Comprehensive Evaluation of Two Kinds of Repair Agents for Gun Tubes
}

\author{
Men Xiang-Dong ${ }^{1, a}$, Tao Feng-He $\mathrm{H}^{1, \mathrm{~b}}$ and Gan $\mathrm{Lin}^{2, \mathrm{c}}$ \\ ${ }^{1}$ Mechanical Engineering College, Shijiazhuang 050003, China; \\ ${ }^{2}$ PLA University of Science and Technology, Nanjing 210007, China \\ àenxd1990@163.com, Fhtao63@126.com, 'ganlin72@163.com
}

Keywords: gun tube, life prolong, repair agent, SAAP, self friction repair agent.

\begin{abstract}
Erosion and wear of the gun tube are the main factors to reduce life of artillery, which restrict the development of the artillery. Gun tube repair agent technology is one of the main methods to reduce the erosion and wear, and extend the life of the tube. Currently, there are main types of repair agent, one is the self adaptive active protection (SAAP) chamber repair agent, and the other is self friction repair agent. Two repair agents are summarized respectively from two aspects, the composition and principle. The repair ability and the scope of application of the two are contrasted and analyzed.
\end{abstract}

\section{Introduction}

Cannon gun tube is expensive, and its purchase expense is about $70 \%$ of fire system purchase expense, in the artillery system. Cannon cannot serve once the tube scrapped. The ablation wear and metal fatigue are two decisive factors of barrel life. At present, the electroslag refining technology used in the steel smelting, basically solved the low fatigue life problem, caused by high chamber pressure and weight requirements of gun tube. Autofrettage technology makes the barrel of the fatigue life further improved [1,2]. Barrel ablation wear life, by contrast, is quite prominent, which has become the main factor of cannon gun tube life. Therefore, the focus to improve the barrel life is currently on the surface of the bore ablation wear problem.

Measures to reduce the erosion and wear mainly include: improved propellant, composite barrel technology, bore surface treatment technology and repair agent technology [3,4]. By joining moderators in propellant or combustible cartridge, the calorific value of the propellant is reduced to increase gun tube life [5]. Composite barrel refers to a technology of manufacturing epoxy resin, carbon fiber and glass fiber coatings on the outside of the ceramic lined, which has been used in the small-bore artillery [6]. In recent years, some scholars began to research preparation chromium coating, tantalum, molybdenum and rhenium in barrel internal, using the explosion welding, electric spark deposition, magnetron sputtering technology, which was still in its infancy

Repair agent technology is to spray the repair agent on bearing band or to wipe bore surface during shooting. Currently, there are mainly two kinds of repair agent working for gun tube protection: one is the self adaptive active protection protecting (SAAP) agent, the other one is self friction repair agent.

\section{Self Adaptive Active Protection (SAAP) Protecting Agent}

Adaptive Active Protection (Self - Adaptive \& Active Protection, hereinafter referred to as the SAAP) technology, is developed by professor Yu. Basic principle is the application of friction and surface modification of chemical theory, which is making rare earth nanometer composite deposit on the substrate surface (including the surface of the cracks occurring in the process of manufacture), and participating in the matrix and crack on the free surface of complex chemical reactions, such as elements of catalytic penetration under the gun and automatic weapon launch process of high temperature, high pressure and frictional chemical conditions, to promote the formation and combining metal ceramic density gradient function of modified layer or aggregates, 
and improve the matrix of high temperature resistant, chemical gas corrosion resistance, anti-friction and anti-wear ability, to strengthen the substrate surface toughness, stop micro cracks growing and make it heal, significantly reduce the erosion wear rate, in order to achieve a full life cycle of active protection, extend the life of the gun tube.

Composition of SAAP. SAAP materials formula is made from high strength, promote and catalytic and coordination of materials, in the processing technology of the mechanical and chemical methods, through organic carrier and auxiliary modification and modification of surface active agent, make powder composite and different components according to certain proportion that grain refinement to $30 \mathrm{~nm}$ to $120 \mathrm{~nm}$, forming SAAP protective material.

High strength phase should be: $\mathrm{SiO}_{2}$-based nonmetal ceramic powders, with particle size 200 or higher, to improve the strength of the modified surface layer;

Promotion phase should be: $\mathrm{Fe}_{2} \mathrm{O}_{3}, \mathrm{Al}_{2} \mathrm{O}_{3}, \mathrm{CaO}$ metal ceramic powders, with particle size 200 mesh or higher;

Catalytic and coordination phase should be: light rare earth metal and oxide series, including La, $\mathrm{Ce}, \mathrm{Pr}, \mathrm{Nd}$, etc., with the initial raw material for average 2000 mesh size, purity of $99.9 \%$;

Carrier phase should be: hydrogenation 26\# white oil or refined base oil;

Coupling agent or surface modification agent phase should be: $\mathrm{N}$ base coupling agent or other good composite effect of surface modification agent, or a mixture of a variety of surface modification agents in proportion.

The mechanism of SAAP. The main cause of erosion wear is the micro crack's growth, expansion and penetration. Mechanism of SAAP technology to solve the above problems is as follows:

1) SAAP can repair the micro-crack, and prevent micro cracks, and block heat channel.

From the dissection and detection of gun tube after live-fire shooting, there are more aggregates in the big cracks on the surface and the furrow pit area of the gun tube after SAAP technical processing, which are products of high temperature sintering of SAAP materials. These aggregates block part of the space between the big cracks and furrows (as shown in figure 1). When high temperature gas carrying huge quantity of heat is prevented from flowing through hot flow channel, the heat absorption rate of the heat channel will not increase as it is close to the tube material on the surface of the substrate. Therefore, the local overheating can be avoided, and the probability of ablation can be reduced.

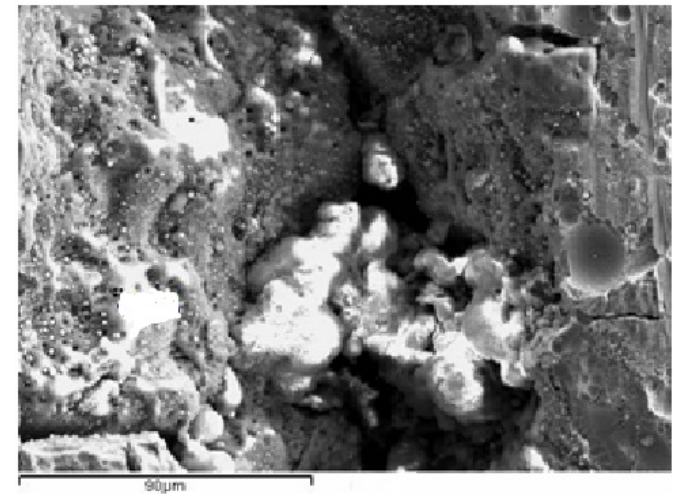

Fig. 1 SAAP block the heat channel of the micro cracks

2) SAAP can alleviate the uneven surface stress and passivate micro cracks.

SAAP material applies to a tube substrate surface. A direct result of the rare earth elements in the nanoscale modification, and the activity of the nanoscale, makes it easy to adsorb and uniformly cover the micro cracks. under the effect of high temperature and high pressure, the rare earths catalytic penetrate in the substrate surface, and form a modified layer that has a certain binding force near the crack, the existence of the modified layer changes the stress distribution around the cracks, and resist the micro crack extension, so as to reduce the uneven phenomenon of the stress around micro cracks, for the passivation of micro cracks.

Figure 2 shows that these rare earth nanomaterials form the needle crystal phase under the condition of certain high temperature (2000 K) and high pressure (about 100-200 MPa). The 
whiskers inlaid around the surface crack reinforce the crack and improve the stress expanding coefficient of crack, and then increases the toughness.

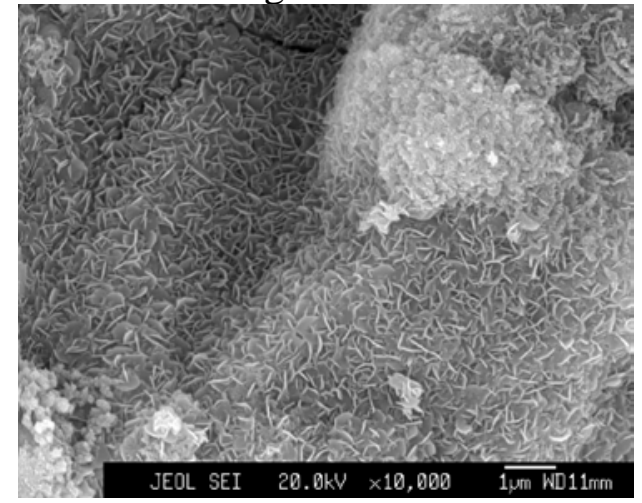

Fig. 2 morphology of SAAP material's distribution around the micro crack

\section{Friction Self-Healing Repair Agent}

Representative is the friction self-healing repair agent produced by Ukrainian XADO Company. Among them, two artillery friction self-healing repair agents code-named PBC I and PBC II are composed of a variety of dispersion of extremely fine mineral substances, in which a variety of materials and catalysts are added. They are used on the surface of the tube and the projectile.

In the process of interior movement, bearing band squeezes repair agent to diffuse into bore surface, similar to the surface carburizing or nitriding process. At contact under the action of high temperature and high pressure, particle "fusion" to the internal lattice of metal surface layer, so as to change the crystal structure of the metal itself. At the same time, the micro rolling dips, repair material, metal particles and other materials involved in friction formed metal ceramic layer structure on the metal friction surface under the synthesis hardening effect, namely the surface repair modification of the wear and tear on metal.

Composition of the Friction Self-Healing Repair Agent. According to the analysis of the relevant references, friction self-healing agent is manufactured according to certain proportion after high-energy ball mill processing on the basis of lamellar silicate mineral material materials, such as talc, serpentine and rare earth oxides. Particle size of common components is 0.1 to $10 \mu \mathrm{m}$ with roughly a quarter of grains' diameter below $1 \mu \mathrm{m}$. Generally organic silicone resin is used as carrier, added into all types of lubricating oils or greases.

The mechanism of the Friction Self-Healing Repair Agent. In the friction process, energy and materials exchange between friction pair and lubrication material, under the effect of mechanical friction, chemical friction and electrochemical friction. What exist on the friction surface in mechanical gradient are metal protective film, metal oxide film, organic polymer film, physical or chemical adsorption film, etc. These films compensate for the wear and corrosion of the friction pair, forming wear self-healing effect. Friction film self-healing is, in fact, a kind of condition self-healing. The existence of relative movement between friction pair and lubricating materials (additive) is the necessary condition to produce self-healing. Self-healing film produces both anti-wear anti-friction effect and compensating the wear effect.

\section{Conclusions}

SAAP materials is nanoscale material, friction bore with self-healing protection agent is micron scale material. These two materials are used in the rare earth materials and on the carrier of lubricating oil. Friction self-healing repair agent is a kind of enhanced bore wear-resisting performance of the chamber protecting agent, and the high temperature erosion is the main reason for the failure of the gun tube. The matrix grease of friction self-healing repair agent, to some extent, can slow down the erosion under the condition of high temperature. Friction self-healing repair agent can fill cracks with a width of 0.1-0.8 mm, and delay gun bore further erosion. SAAP material 
is a kind of nano materials which can penetrate into the tiny crack of the bore. Sintering mass through to can retard the further erosion by preventing the high temperature heat flux. SAAP have very good protection effect repairing gun bore cracks with the width below $0.1 \mathrm{~mm}$.

\section{References}

[1]Y H Li, A theoretical study on the swage autofrettage of gun tubes. Acta. Armamentarii. 01(1995) $1-7$.

[2]Q Z Kong, Y L Li, S H Luo, The production and practice of large caliber gun tube before the autofrettage process. Mat. Pro. 03(2000)34-35.

[3]Y C Chen, Q Z Song, J Z Wang, New Technologies to Extend the Erosion Life of Gun Barrel. Acta. Armamentarii. 02(2006)330-334.

[4]L Gan, F H Tao, X H Lu, J J Zhou, Study of Cannon gun tube life prolong. J. Artill. L\&Contr. 03(2006)10-14.

[5]H G Li, J Yan, S G Du, H P Cui, Review: cannon gun tube corrosion inhibitor technology. J. Artill. L\&Contr. 4(2012) 103-106

[6]L M Chen, L F Qian, Study on Erosion and Service Life of Composite Material Barrel. J. Artill. L\&Contr. 06(2005)89-93. 